OPEN ACCESS

Edited by:

Tao Xu,

Anhui Medical University, China

Reviewed by:

Dapeng Chen,

Dalian Medical University, China

Rosanna Di Paola

University of Messina, Italy

${ }^{*}$ Correspondence:

Yuanyuan Wang

shetao12201@126.com

Specialty section: This article was submitted to Gastrointestinal and Hepatic Pharmacology, a section of the journal Frontiers in Pharmacology

Received: 17 April 2021 Accepted: 14 June 2021

Published: 16 July 2021

Citation:

Mei C, Wang X, Meng F, Zhang X,

Chen L, Yan S, Xue J, Sun $X$ and Wang $Y$ (2021) Aucuboside Inhibits the

Generation of Th17 Cells in

Mice Colitis.

Front. Pharmacol. 12:696599. doi: 10.3389/fphar.2021.696599

\section{Aucuboside Inhibits the Generation of Th17 Cells in Mice Colitis}

\author{
Chenxue Mei ${ }^{1,2}$, Xiao Wang ${ }^{1}$, Fanxiang Meng ${ }^{1}$, Xiaoqing Zhang ${ }^{1}$, Ling Chen ${ }^{3}$, Siqi Yan ${ }^{1}$, \\ Junxiu Xue ${ }^{1}$, Xun Sun ${ }^{1}$ and Yuanyuan Wang ${ }^{4 *}$ \\ ${ }^{1}$ Department of Immunology, College of Basic Medical Sciences of China Medical University, Shenyang, China, ${ }^{2}$ Department of \\ Gastroenterology, Shengjing Hospital of China Medical University, Shenyang, China, ${ }^{3}$ Department of Gastroenterology, Jinqiu \\ Hospital of Liaoning Province, Shenyang, China, ${ }^{4}$ Department of Anesthesiology, The Fourth Affiliated Hospital, China Medical \\ University, Shenyang, China
}

Aucuboside is an iridoid glycoside extracted from traditional Chinese medicine such as Rehmannia glutinosa, possessing a wide range of biological activities, including antioxidant, anti-aging, anti-inflammatory, and anti-fibrotic effects. The effects of aucuboside on inflammatory bowel disease (IBD) have not been studied. Therefore, the effects of aucuboside on the generation of Foxp3+ regulatory $T$ (Treg) cells and IL17-producing T helper (Th17) cells in colitis were studied. A mouse colitis model was established by intracolonic administration of 2,4,6-trinitrobenzene sulfonic acid (TNBS) to mimic human IBD. The generation of Treg and Th17 cells was evaluated by flow cytometry. Aucuboside significantly alleviated colitis symptoms, including weight loss, high disease activity index, and inflammatory responses. The generation of Th17 cells in colitis was significantly inhibited by aucuboside and accompanied by the suppression of IL-17 expression. In Raw264.7 cells, the LPS-induced increase in IL-17 expression was also suppressed by aucuboside, which was significantly blocked by the ROR $\gamma$ t inhibitor sr2211. In addition, the decrease in the proportion of Treg cells was also partially reversed by aucuboside, which may reflect the aucuboside-induced inhibition of Th17 cells. This previously unrecognized immunoregulatory function of aucuboside may have clinical applications in IBD.

Keywords: aucuboside, inflammation, Treg, Th17, colitis

\section{INTRODUCTION}

Inflammatory bowel disease (IBD) is an inflammatory disease of the colon with multifactorial etiology, including ulcerative colitis (UC) and Crohn's disease (CD). IBD is characterized by periodic remission and deterioration (McDowell and Haseeb, 2019). The incidence of IBD worldwide varies between 0.5 and 24.5 per 100,000 people, while in China, the occurrence rate of UC is 11.6 and that of CD is 1.4 per 100,000 people (Sugimoto et al., 2008). IBD promotes significant gastrointestinal symptoms, such as bloody diarrhea, abdominal pain, anemia, and weight loss (Mateus et al., 2018; Siracusa et al., 2020). Fibrosis, obstruction, and cancer are complications of IBD that often affect the quality of life of patients (Zhang et al., 2021). Current medical therapies such as corticosteroids and immunomodulators are still limited by some severe side effects and complications. Therefore, it is still necessary to discover some more novel pharmacologic approaches.

The pathogenesis of IBD is multifactorial, with increasing evidence suggesting an important role in the balance between regulatory T cells (Treg) and Type $17 \mathrm{~T}$ helper cells (Th17) (Geremia et al., 2014). Th17, a subtype of CD4+ T cells, are pro-inflammatory $\mathrm{T}$ helper cells that play a dominant 
A<smiles>OCC1=C[C@@H](O)[C@@H]2C=CO[C@H](O[C@@H]3O[C@H](CO)[C@@H](O)C[C@H]3O)[C@H]12</smiles>

D

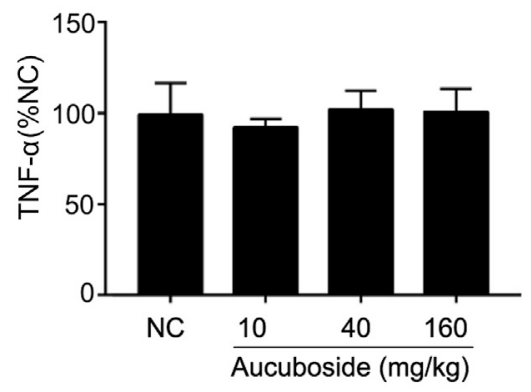

B

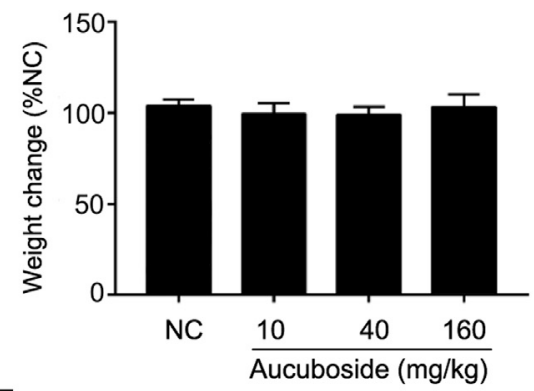

E

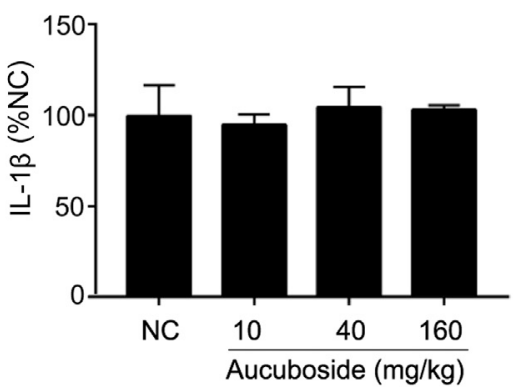

C

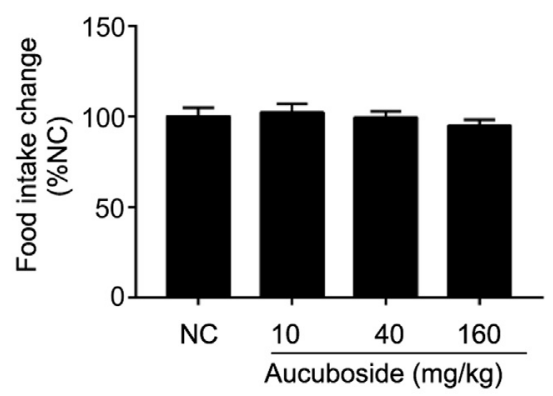

F

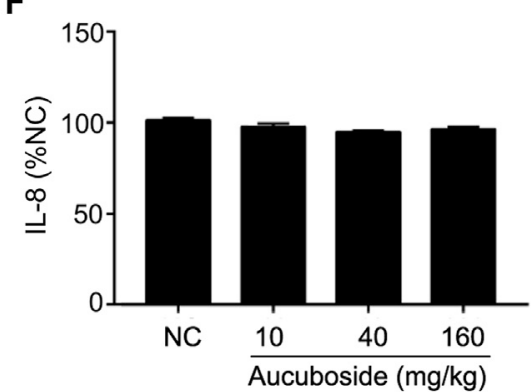

FIGURE 1 | Effects of aucuboside on normal mice, toxicology of arbutin in vitro and in vivo, and chemical structure of aucuboside (A). Effects of gavage administration of aucuboside on body weight change (B), food intake (C), and colonic expression of TNF- $\alpha$, IL-1 $\beta$, and IL-8 (D-F). Data are expressed as mean \pm SD. Values in the normal control (NC) group are 100\%, and other data are given relative to the NC value.

role in the production of pro-inflammatory cytokines, such as interleukin (IL)-17, IL-21, and IL-22, which serve as markers of inflammation (Nakae et al., 2003). Excessive IL-17 secreted by Th17 would recruit neutrophils and monocytes and increase the production of TNF- $\alpha$ and IL- $1 \beta$, contributing to tissue damage and exacerbating inflammation in the gut (Kolls and Lindén, 2004; Korn et al., 2009). Another subtype of CD4+ T cells, Tregs, are immunosuppressive cells that participate in the regulation of colitis progression (Cao et al., 2004). Intercellular interactions and the secretion of IL-10 are the main pathways by which Tregs can regulate the inflammatory response (Rothstein and Camirand, 2015).

It is factually beneficial to uncover potential drugs/ compounds to regulate the generation of Tregs in the treatment of IBD. Zhang et al. firstly reported that D-mannose, a C-2 epimer of glucose, stimulates Treg cell differentiation in human and mouse cells. Both aucuboside and D-mannose can be isolated from Lathraea squamaria L. (Swiatek and Dombrowicz, 1976); however, the effects of aucuboside on the generation of Tregs/Th17 cells were not studied. This study aimed to explore the effects of aucuboside on the generation of Tregs/Th17 cells in colitis.

Aucuboside used in this study was isolated from the traditional Chinese medicinal plant Eucommia ulmoides. Eucommia ulmoides exerts a wide range of biological activities including antioxidant, anti-aging, antiinflammatory, and anti-fibrotic effects (Murakami et al., 2018). In this study, the effects of aucuboside on Treg/Th17 balance and aucuboside-induced Treg/Th17 balance in colitis were investigated. A mouse colitis model was established using intracolonic administration of 2,4,6-trinitrobenzene sulfonic acid (TNBS) to mimic human IBD.

\section{MATERIALS AND METHODS}

\section{Reagents}

Aucuboside (purity $\geq 98 \%$ ) was obtained from Chengdu Pusi Biotechnology Co., Ltd. (Chengdu, China). Sulfasalazine (SASP) was purchased from Tianjin Kingyork Group Co., Ltd. (Tianjin, China). Antibodies to iNOS (ab110304), p-p65 (ab62484), cleaved caspase 3 (cl-caspase3, ab13847), Foxp3 (ab21685), and ROR $\gamma \mathrm{t}$ (ab59348) were purchased from Abcam (Hong Kong) Ltd. (Hong Kong, China). Chemicals were obtained from Sigma-Aldrich (St. Louis, MO, United States) unless otherwise indicated.

\section{Animals and Experiment Design}

The experimental protocol was approved by the China Medical University Animal Care and Ethics Committee (CMU2019218), SYXK (Liao) 2018-0008. Thirty male SPF C57BL/6 mice of 6-8 weeks of age were acclimatized to laboratory conditions $\left(23^{\circ} \mathrm{C}, 12 \mathrm{~h} / 12 \mathrm{~h}\right.$ light/dark, $50 \%$ humidity, and ad libitum access to food and water) for 2 weeks before the experiments. The animal protocol was designed to minimize pain and discomfort to the animals. 


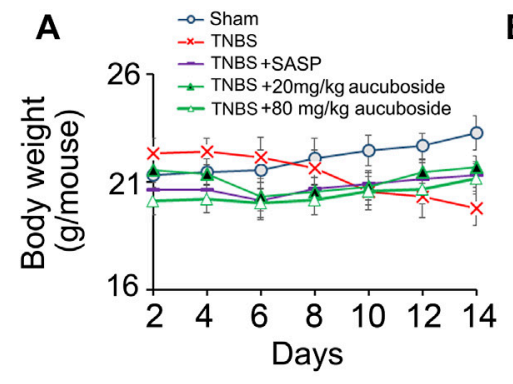

D

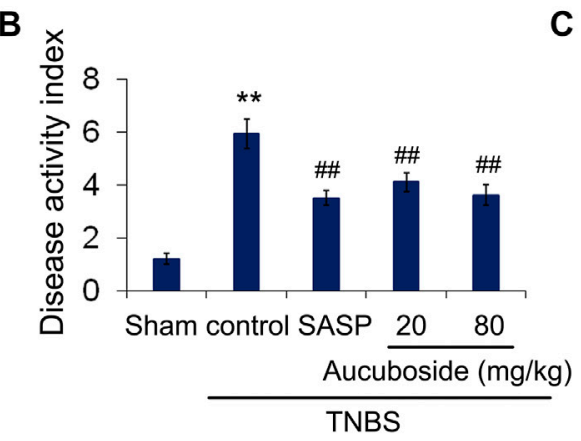

TNBS

C

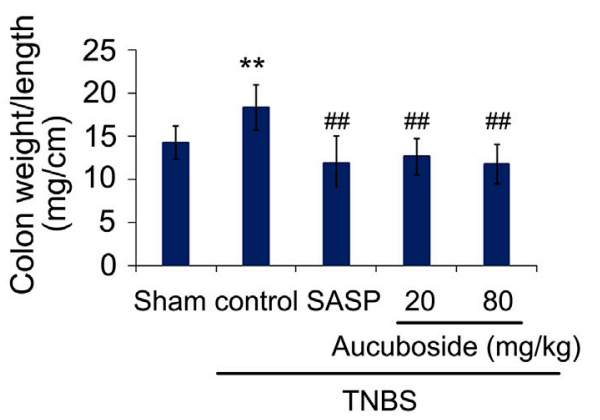

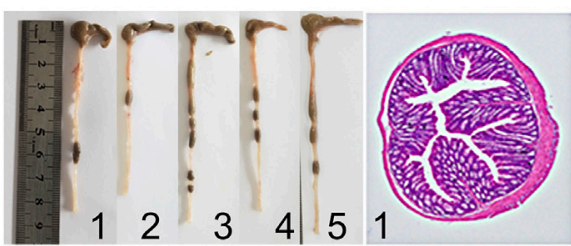

Sham

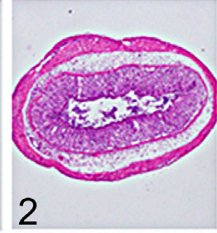

control

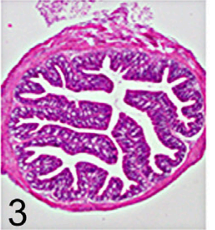

SASP
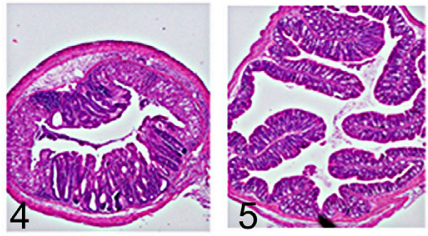

$\frac{20}{\text { Aucuboside }(\mathrm{mg} / \mathrm{kg})}$

TNBS

FIGURE 2 |Effects of aucuboside on colitis symptoms. After 13 days of treatment, the effects of aucuboside on body weight loss (A), disease activity index (B), and colon weight-to-length ratio (C) were evaluated. The macroscopic observation and hematoxylin-eosin-stained colon sections (scale bar = $100 \mu \mathrm{m})$ (D) are also shown. Data are expressed as mean \pm SD. Values in the sham group are $100 \%$, and other data are given relative to the sham group. ${ }^{* *} p<0.01$ compared with the sham group; $\# \# p<0.01$ compared to the colitis control; $n=6$ mice.

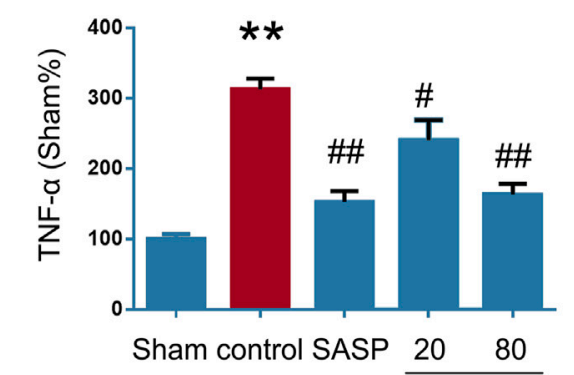

Aucuboside $(\mathrm{mg} / \mathrm{kg})$

TNBS

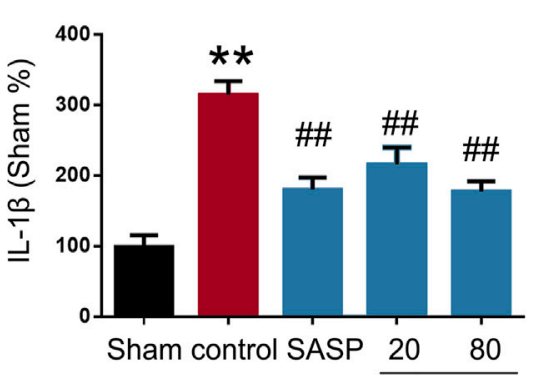

Aucuboside $(\mathrm{mg} / \mathrm{kg})$

TNBS

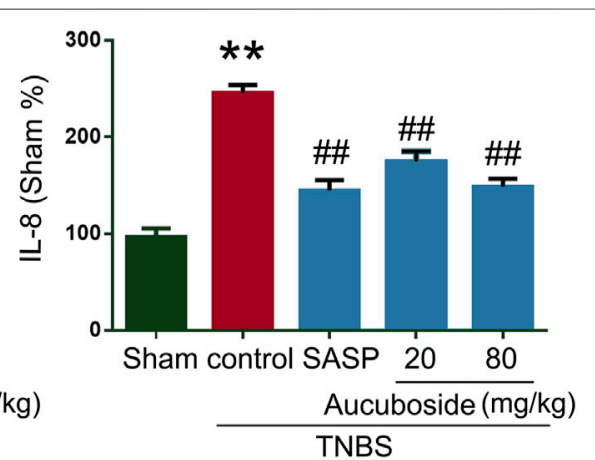

TNBS

FIGURE 3 | Effects of aucuboside on cytokine expression. After 13 days of aucuboside treatment, the colonic expressions of TNF- $\alpha$, IL-1 $\beta$, and IL-8 (A-C) were measured. Data are expressed as mean $\pm \mathrm{SD}$. ${ }^{\star \star} p<0.01$ compared with the sham group; ${ }^{\#} p<0.05$, ${ }^{\# \#} p<0.01$ compared with the colitis control; $n=6$ mice.

Mice were housed one per cage and were deprived of food for $12 \mathrm{~h}$ before the experiments.

After adaptive feeding for one week, 60 mice were randomly divided into six groups ( $n=6 /$ group): group I, sham-operated control with intracolonic administration of saline; group II, colitis group; and groups III, IV, and V treated with SASP (100 $\mathrm{mg} / \mathrm{kg}$ body weight, intragastric, dissolved in saline), lowdose aucuboside $(20 \mathrm{mg} / \mathrm{kg}$ body weight, intragastric, dissolved in saline), and high-dose aucuboside $(80 \mathrm{mg} / \mathrm{kg}$ body weight, intragastric, dissolved in saline), respectively, 1 day after the induction of colitis. SASP and aucuboside were administered by gavage once per day for 14 consecutive days.
The mice colitis model was induced as described previously (Xiong et al., 2016; Mateus et al., 2018). Briefly, mice were fasted for $24 \mathrm{~h}$ with free access to drinking water. A catheter was inserted through the anus to the approximate level of the splenic flexure under urethane anesthesia. The colon was then infused with $100 \mu \mathrm{L}$ TNBS dissolved in ethanol (50\% v/v). The mice were allowed to eat and drink ad libitum from $1 \mathrm{~h}$ after the operation. Distal colon samples from the full-thickness intestinal wall were harvested for biochemical studies. After 14 days, blood samples were collected from the eye, and then mice were sacrificed by cervical dislocation. The spleen and mesenteric lymph nodes were collected, and colon tissue 
A

(kDa)
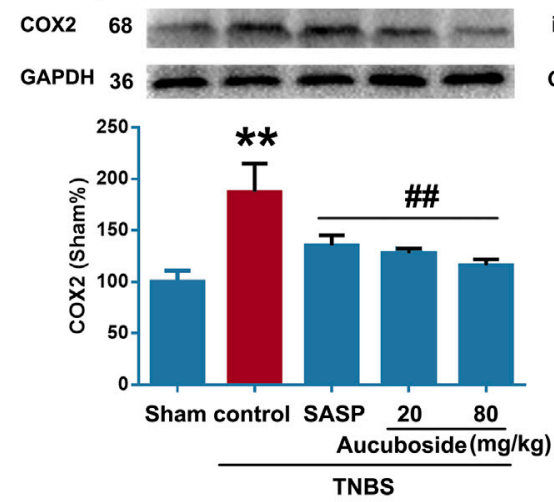

D

(kDa)
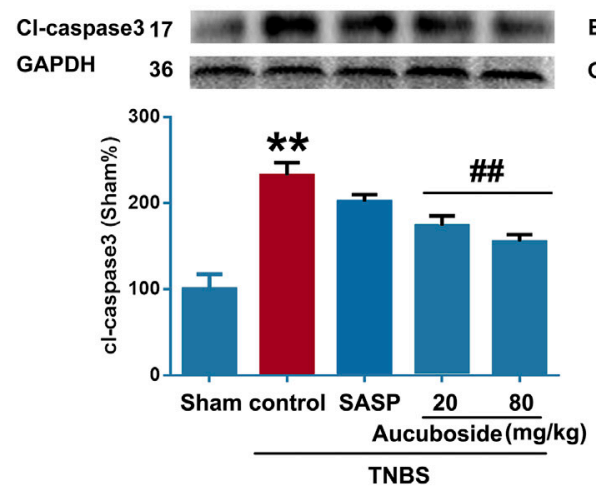

B

(kDa)
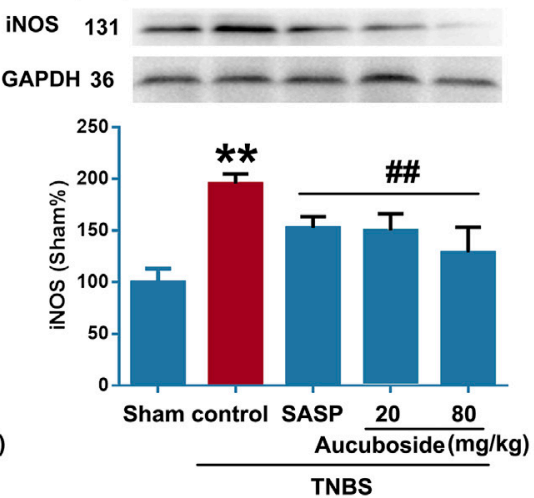

E

(kDa)

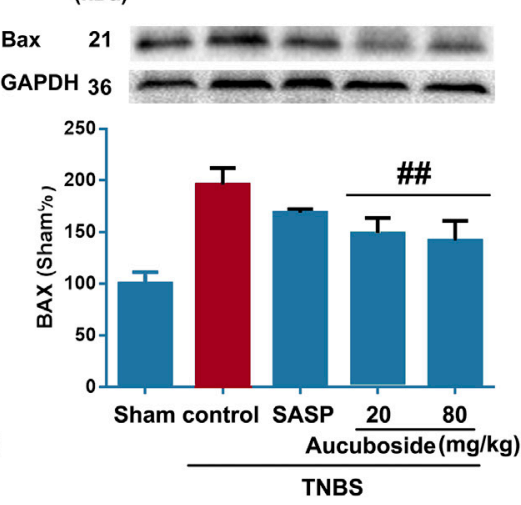

C

(kDa)
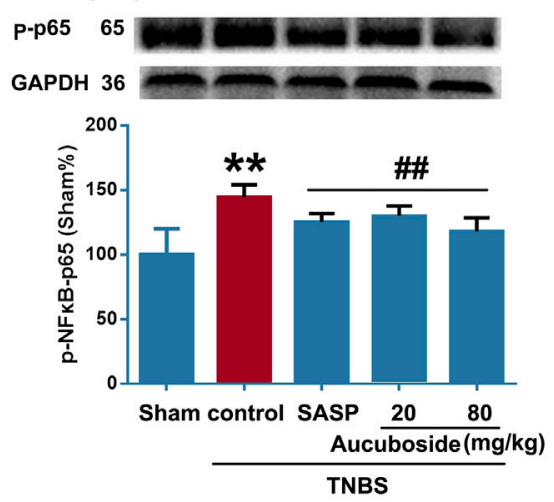

$\mathbf{F}$
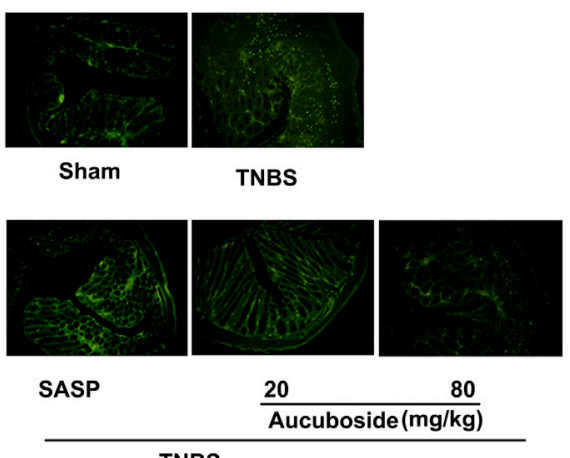

TNBS

FIGURE 4 | Effects of aucuboside on inflammation and apoptosis in colitis. After 13 days of aucuboside treatment, effects on colonic inflammatory mediators (A) COX-2, (B) iNOS, and (C) phosphorylated NF-kB-p65 (p-p65) were studied by western blotting. The effects of aucuboside on apoptosis-related proteins (D) cleaved caspase 3 (Cl-caspase3) and (E) Bax were studied by western blotting. (F) TUNEL was used to test the effects of aucuboside on epithelial cell apoptosis in colitis. Data are expressed as mean \pm SD. Values in the sham group are $100 \%$, and other data are given relative to the sham group. ${ }^{* *} p<0.01$ compared with the sham group; ${ }^{\# \#} p<0.01$ compared with the colitis control; $n=6$ mice.

samples were retained and preserved. Body weight, food intake, and defecation were recorded daily. The disease activity index and the colon weight/length ratio were determined. Colon histology, morphology, and the severity of inflammation were assessed in hematoxylin and eosin (HE)stained colon sections. Harvested colon tissue specimens were cut into $5 \mathrm{~mm}$ pieces, processed, and mounted on slides for immunohistochemical and western blot analyses.

\section{Treg/Th17 Analysis by Flow Cytometry}

Fresh spleen and mesenteric lymph nodes were placed in $3 \mathrm{ml}$ PBS containing $10 \%$ FBS and ground for 2 min on a 200 -mesh nylon sieve using a $5 \mathrm{ml}$ syringe piston and transferred to $15 \mathrm{ml}$ EP tubes. The spleen tissue homogenate was centrifuged at $1,100 \mathrm{r} / \mathrm{min}$ for $10 \mathrm{~min}$, and the deposited cells were retained. RBC lysate $(2 \mathrm{ml}$; Beijing Solarbio Science \& Technology Co., Ltd., Beijing, China) was added and incubated under dark conditions for $15 \mathrm{~min}$ and then centrifuged at $2,240 \mathrm{r} / \mathrm{min}$ for $5 \mathrm{~min}$. After washing with $10 \mathrm{ml}$ RPMI-1640 solution, a single lymphocyte cell suspension was obtained. The mesenteric lymph node homogenate was centrifuged at $2,000 \mathrm{r} / \mathrm{min}$ for $5 \mathrm{~min}$; the deposited cells were retained; and after washing with $10 \mathrm{ml}$ RPMI-1640 solution, a single lymphocyte cell suspension was obtained. The prepared single-cell suspension was transferred to a $1.5 \mathrm{ml}$ EP tube by secondary filtration using a 200-mesh nylon sieve, and the cell number was adjusted to $1 \times 10^{6} / \mathrm{ml}$.

To detect the number of Tregs, collected cells were incubated with $2 \mu \mathrm{L}$ pre-chilled anti-mouse CD4 and CD25 antibodies (BD Biosciences, United States) and fixed at $4^{\circ} \mathrm{C}$ in a dark room for $30 \mathrm{~min}$. After washing with PBS containing 10\% FBS and centrifuging at $1,200 \mathrm{r} / \mathrm{min}$ for $6 \mathrm{~min}$ at $4^{\circ} \mathrm{C}$, cell membrane breaking lysate (BD Biosciences) was added to the cell suspension, avoiding light, and incubated for hours. Then, $2 \mu \mathrm{L}$ pre-chilled anti-mouse factor Foxp3 antibody (BD Biosciences) was added to the collected cells and fixed at $4^{\circ} \mathrm{C}$ in a dark room for $30 \mathrm{~min}$. To detect the number of Th17 cells, PMA $(30 \mathrm{ng} / \mathrm{ml})$, ionomycin $(1 \mu \mathrm{g} / \mathrm{ml})$, and BFA $(10 \mathrm{ng} / \mathrm{ml})$ were added to the collected cells and incubated for $4 \mathrm{~h}$ before 


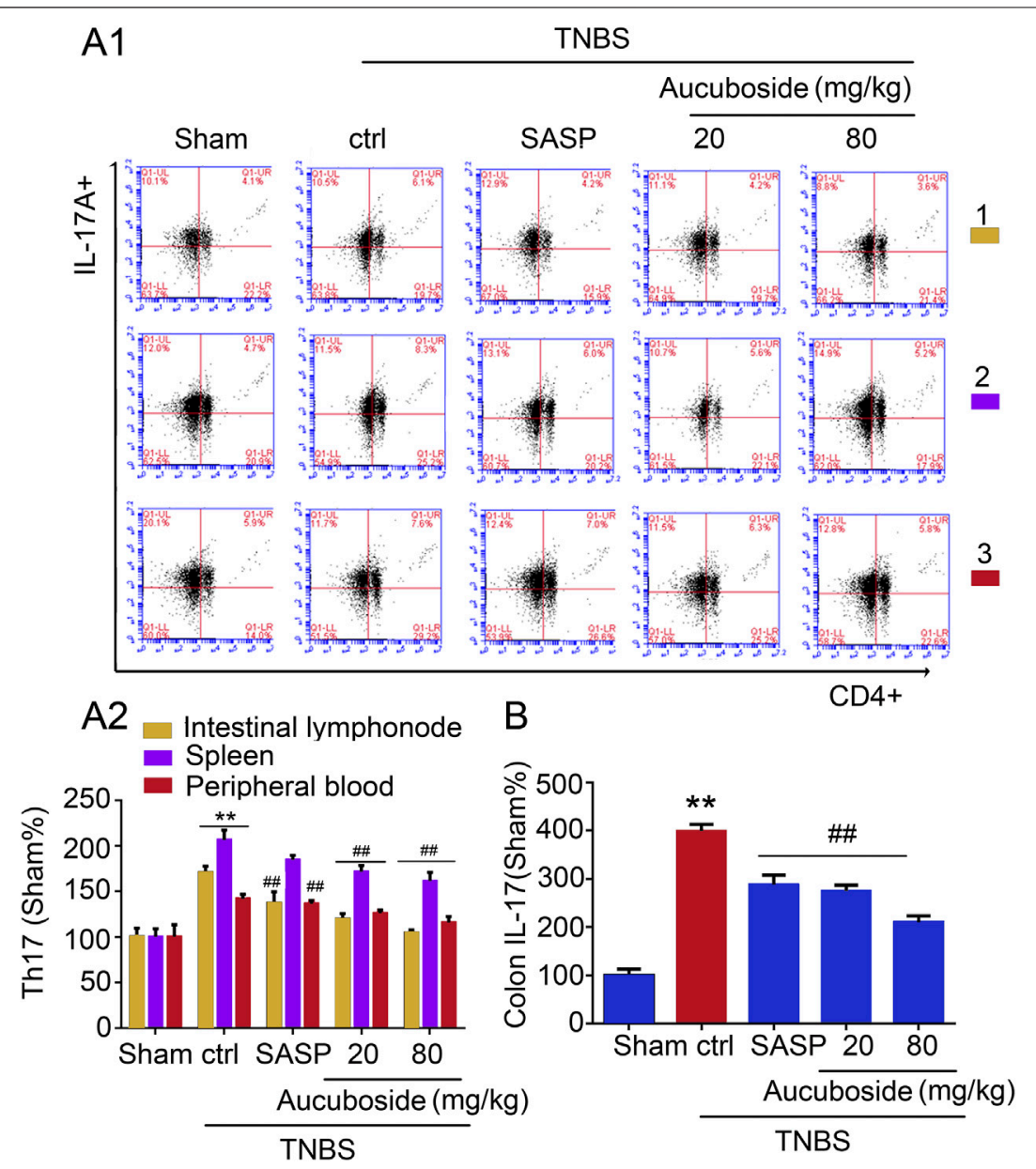

FIGURE 5 | Effects of aucuboside on the generation of Th17 cells. (A) (i) and (ii) are the representative image and statistical analysis of the generation of Th17 cells in colonic mesenteric lymph nodes (1), spleen (2), and peripheral blood (3), respectively. (B) Effects of aucuboside on release of IL-17. Values in the sham group are $100 \%$, and other data are given relative to the sham group. ${ }^{\star \star} p<0.01$ compared with the sham group; ${ }^{\# \#} p<0.01$ compared with the colitis control; $n=6$ mice.

filtering and adjusting the cell number. The staining of Th17 cells using FITC anti-mouse CD4 and APC anti-mouse IL-17A followed the same procedure as Tregs. After washing, the cells were resuspended in $200 \mu \mathrm{L}$ PBS containing 10\% FBS for flow cytometry analysis.

\section{Quantitative Real-Time Polymerase Chain Reaction}

RNA isolation was carried out using Trizol (Takara, Dalian, China) per the manufacturer's instructions. Total RNA was quantified using a NanoDrop instrument to measure optical density (OD) at $260 / 230 \mathrm{~nm}$, and purity was evaluated by obtaining the absorbance ratio at $260 / 280 \mathrm{~nm}$. cDNA was synthesized from $1 \mu \mathrm{g}$ of total RNA with a Thermo Scientific Verso cDNA Kit according to the manufacturer's instructions. Quantitative real-time PCR (qRT-PCR) was performed using 10-20 ng of cDNA with SYBR green master mix (Takara, United States) and a StepOnePlus RTPCR system (Applied Biosystems, Inc., United States). The differences in mRNA expression at all the gene levels were calculated as the fold change using the formula 2- $\Delta \Delta \mathrm{ct}$. Primer sequences for Foxp 3 were as follows: Foxp3 forward: $5^{\prime}$-TGG CTCCAAGGATGGTTAGC-3' and Foxp3 reverse: $5^{\prime}$ - TCA GGGACAGGGTTGACAGT-3' (provided by General Biosystems). Primer sequences for ROR $\gamma \mathrm{T}$ were as follows: ROR $\gamma \mathrm{T}$ forward: 5'-TCCATATTTGACTTTTCCCACT-3' and ROR $\gamma \mathrm{T}$ reverse: 5'-GATGTTCCACTCTCCTCTTCTC$3^{\prime}$. As a control, mRNA content for GAPDH was analyzed using the following primers: GAPDH forward: $5^{\prime}$-GCCACC CAGAAGACTGTGGAT- $3^{\prime}$ and GAPDH reverse: 5'-GGA AGGCCATGCCAGTGA-3' (provided by General Biosystems).

\section{Statistical Analysis}

GraphPad Prism (version 9.0; GraphPad Prism Software, Inc., La Jolla, CA, United States) was used to perform statistical analysis. A non-parametric test, one-way ANOVA, was used for data comparisons between three or more groups, followed 


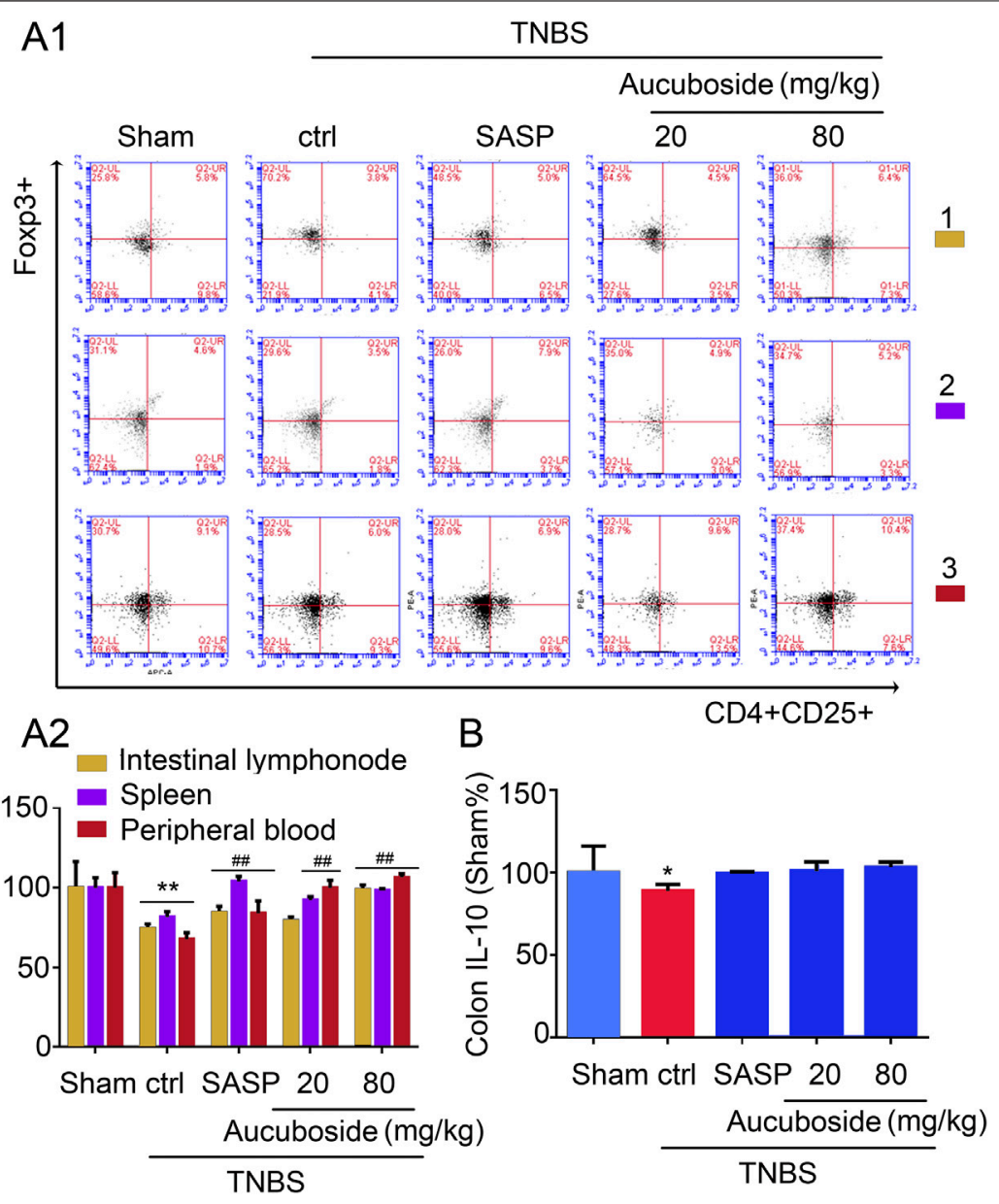

FIGURE 6 | Effects of aucuboside on the generation of Treg cells. (A) (i) and (ii) are the representative image and statistical analysis of the generation of Treg cells in colonic mesenteric lymph nodes (1), spleen (2), and peripheral blood (3), respectively. (B) Effects of aucuboside on release of IL-10. Values in the sham group are 100\%, and other data are given relative to the sham group. ${ }^{\star} p<0.05,{ }^{\star \star} p<0.01$ compared with the sham group; ${ }^{\# \#} p<0.01$ compared with the colitis control; $n=6$ mice.

by the Kruskal-Wallis rank-sum test. All results were obtained from at least three independent experiments.

\section{RESULTS}

\section{Effects of Aucuboside on Normal Mice}

The chemical structure of aucuboside is shown in Figure 1A. For normal control mice, the gavage administration of 10,40 , and $160 \mathrm{mg} / \mathrm{kg}$ aucuboside consecutively for 14 days did not significantly affect body weight (Figure 1B), food intake (Figure 1C), or colonic pro-inflammatory cytokines, including TNF- $\alpha$ (Figure 1D), IL-1 $\beta$ (Figure 1E), and IL-8 (Figure 1F). Based on these preliminary experiments and previous reports, we selected the maximum dose of aucuboside as $80 \mathrm{mg} / \mathrm{kg}$ in this study.

\section{Aucuboside Ameliorated Colitis Symptoms} Compared with the sham group, body weight loss (Figure 2A), higher disease activity index (Figure 2B), and an increased colon weight-to-length ratio (Figure $\mathbf{2 C}$ ) were observed in the TNBS colitis group. The micromorphology and histomorphology are shown in Figure 2D. Compared with the sham control, destroyed microvilli, incomplete mucosal structure, and infiltration of immune cells were all significant. Similar to the SASP-induced treatment effects, all pathological changes in colitis were significantly reversed by aucuboside.

\section{Aucuboside Alleviated Cytokine Profile in Colitis}

Compared with the sham group, colonic pro-inflammatory cytokines, including TNF- $\alpha$, IL- $1 \beta$, and IL- 8 , were significantly increased in the colitis group, and these changes could be significantly reversed by 20 and $80 \mathrm{mg} / \mathrm{kg}$ aucuboside (Figure 3). These results showed that aucuboside could decrease inflammation in colitis.

\section{Aucuboside Reduced the Release of Inflammatory Mediators and Inhibited Apoptosis}

As shown in Figures $\mathbf{4 A - C}$, colonic epithelial COX-2, iNOS, and phosphorylated NF- $\kappa$ B-p65 (p-p65) were significantly increased 


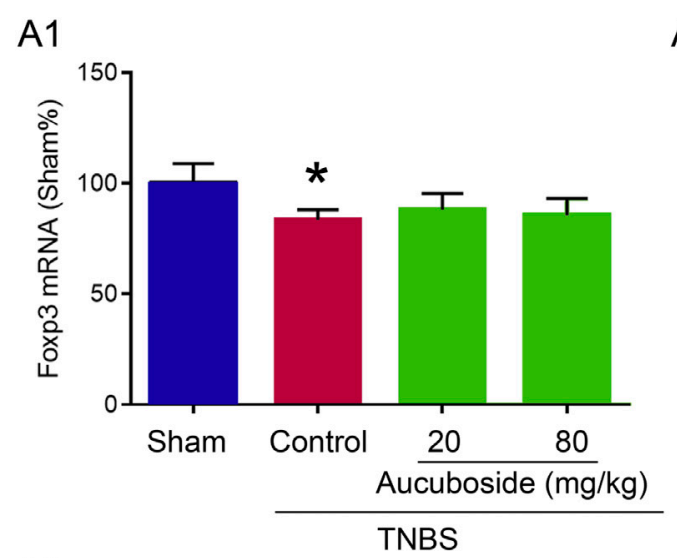

A3

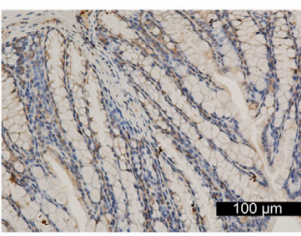

Sham

TNBS
A2
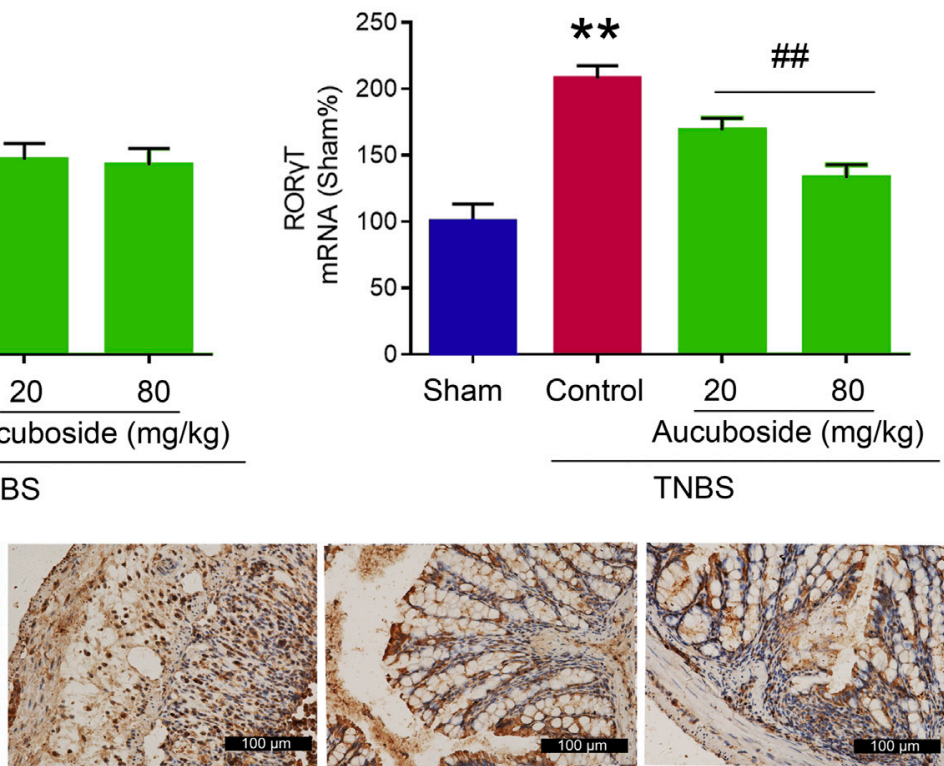

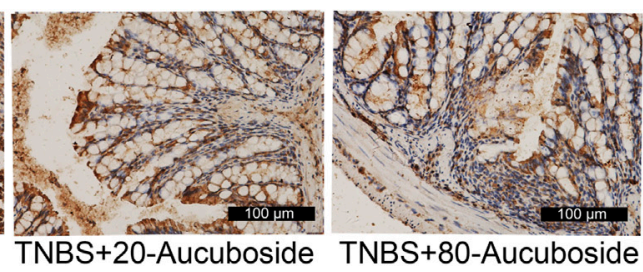

FIGURE 7|Potential mechanism underlying aucuboside-induced regulation of Th17 cells and Treg cells. (A) (i) Effects of aucuboside on Foxp3 mRNA expression and (ii) effects of aucuboside on ROR $\gamma \mathrm{t}$ mRNA expression; (iii) immunohistochemical analysis of ROR $\gamma \mathrm{t}$ expression. ${ }^{*} p<0.05$, ${ }^{\star \star} p<0.01$ compared with sham; ${ }^{* \#} p<$ 0.01 compared with the colitis control; $n=6$ independent experiments.

compared with the sham control; both SASP and aucuboside significantly reversed these changes. These results further confirmed that aucuboside could exert anti-inflammation effects on colitis.

The apoptosis markers cl-caspase3 (Figure 4D) and Bax (Figure 4E) were significantly increased in the colitis group, which was reversed by 20 and $80 \mathrm{mg} / \mathrm{kg}$ aucuboside. The TUNEL-positive cells in the colonic epithelium of the colitis group were significantly increased compared with the sham group. After treatment with 20 and $80 \mathrm{mg} / \mathrm{kg}$ aucuboside, the TUNEL-positive cells in the colonic epithelium were significantly decreased (Figure 4F). These results suggested that aucuboside has anti-apoptotic effects.

\section{Effects of Aucuboside on the Generation of Th17 Cells}

Compared with the sham group, the generation of Th17 cells in colonic mesenteric lymph nodes, spleen, and peripheral blood was significantly increased in the colitis group (Figures 5Ai,ii). Gavage administration of 20 and $80 \mathrm{mg} / \mathrm{kg}$ aucuboside significantly decreased the proportion of Th17 in the colitis group (Figures 5Ai,ii). IL-17, as an effective cytokine in Th17 cells, was also studied in this study. As shown in Figure 5B, IL-17 was significantly increased in the colitis group, which was significantly reversed by different doses of aucuboside. These results suggest that the inhibition of Th17 production may be involved in the effects on colitis induced by aucuboside.

\section{Effects of Aucuboside on the Generation of Treg Cells}

Compared with the sham group, the Treg cell number in colonic mesenteric lymph nodes, spleen, and peripheral blood was significantly decreased in the colitis group (Figures 6Ai,ii). Gavage administration of 20 and $80 \mathrm{mg} / \mathrm{kg}$ aucuboside significantly increased the Treg cell number in the colitis group (Figures 6Ai,ii). However, IL-10, the effective cytokine of Tregs, was not significantly affected by aucuboside (Figure 6B). These results suggest that an increase in Tregs may not be the main mechanism associated with aucuboside treatment.

\section{Mechanisms Underlying Aucuboside- Induced Inhibition of Th17}

To clarify the mechanisms underlying the aucuboside-induced regulation of Treg and Th17 cell generation, the mRNAs of Foxp3 and ROR $\gamma \mathrm{t}$ were studied. As shown in Figure 7Ai, the colonic Foxp3 mRNA was significantly decreased in the colitis group, which was not significantly affected by 20 and $80 \mathrm{mg} / \mathrm{kg}$ aucuboside. Colonic ROR $\gamma \mathrm{t}$, as the main regulating factor promoting Th17 cell differentiation, was significantly increased in colitis, and the increase was reversed by both 20 and $80 \mathrm{mg} / \mathrm{kg}$ aucuboside (Figures 7Aii,iii). These results suggest that aucuboside may inhibit Th17 cell generation through blocking ROR $\gamma \mathrm{t}$ activity. 


\section{DISCUSSION}

In this study, the effects of aucuboside and underlying mechanisms on mice colitis were studied. Gavage administration of aucuboside significantly alleviated TNBS-induced colitis; the colitis symptoms including weight loss, higher disease activity index, mucosal necrosis, and inflammatory cell infiltration were significantly alleviated by aucuboside. The generation of Th17 cells in colitis was inhibited by aucuboside. The inhibition of Th17 cells is the mechanism involved in the aucuboside-induced treatment effect in colitis.

The expression of inflammatory cytokines and inflammatory mediators in colitis was significantly reduced by aucuboside. These results suggested that aucuboside has an antiinflammation function. It was also shown that aucuboside can inhibit inflammatory responses in experimental traumatic brain injury (Wang et al., 2020). Our study indicated that aucuboside has an anti-inflammatory potential in colitis. Aucuboside administration also significantly inhibited intestinal epithelial cell apoptosis in colitis. All these results suggested that aucuboside can exert protective effects against TNBS-induced colitis in mice.

We focused on the effects of aucuboside on the generation of Treg and Th17 cells because the increase in Th17/Treg is one of the main pathological mechanisms of colitis. Our study showed that the number of Th17 cells in colitis was reduced by aucuboside and the IL-17 expression was also suppressed by aucuboside. Aucuboside-induced inhibition of Th17 is related to the blocking of $\operatorname{ROR} \gamma \mathrm{t}$. $\operatorname{ROR} \gamma \mathrm{t}$ is a major transcription factor that regulates Th17 cell differentiation, and $\mathrm{ROR} \gamma \mathrm{t}$ inhibitors were effective in reducing the severity of experimental autoimmune diseases (Ivanov et al., 2007; Bassolas-Molina et al., 2018). Aucuboside also reversed the decrease in Treg cell number in colitis, but this did not affect the anti-inflammation function. We speculated that the aucuboside-induced activation of Tregs could be a reflection of Th17 inhibition. Further experiments confirmed our supposition because aucuboside did not affect the expression of Foxp3. In the infiltration of inflammatory cells caused by IBD, CD $4+\mathrm{T}$ cells are closely concerned with disease activity and disease progression and associated with pro-inflammatory cytokine levels (Müzes et al., 2012; Smids et al., 2017). The balance of Tregs/Th17 cells is one of the important mechanisms of IBD progression. In the initial differentiation stage, $\mathrm{CD} 4+\mathrm{T}$ cells are activated by the synergistic effect of TGF- $\beta$ and IL- 6 and ROR $\gamma \mathrm{t}$ activation is simultaneously induced, leading to the final differentiation into Th17 cells (Bettelli et al., 2006; Sandquist and Kolls, 2018). ROR $\gamma$ t can also regulate the function of mature Th17 cells, which is necessary for IL-17 generation (Ivanov et al., 2006). In IBD patients, the overactivation of Th17 cells mediated by $\operatorname{ROR} \gamma \mathrm{t}$ and impaired Treg function is often observed, which leads to a break in the balance of Treg and Th17 cells (Yamada et al., 2016; Hou and Bishu, 2020). The extract of traditional Chinese medicine Eucommia can downregulate various pro-inflammatory cytokines, including IL-17, to treat rheumatoid arthritis in rats (Wang et al., 2018). In our experiments, aucuboside extracted from Eucommia could reverse the increase of pro-inflammatory cytokines caused by inflammatory cell infiltration in a colitis model. Aucuboside improves the Treg/Th17 balance and finally leads to the alleviation of TNBS-induced colitis.

There are also some shortcomings in this study. First, the detailed underlying mechanisms of how aucuboside inhibits Th17 differentiation were not studied. According to our current results, it is proper that aucuboside may inhibit the secretion of specific cytokines which inhibits ROR $\gamma \mathrm{t}$ activity, which finally results in the inhibition of the generation of Th17 cells. However, the detailed mechanism needs further study. Second, the binding pattern and interaction mode between aucuboside and $\operatorname{ROR} \gamma \mathrm{t}$ were not elaborated clearly. However, these shortcomings did not affect our main findings. Aucuboside is significantly protective against TNBS-induced colitis; moreover, Th17 inhibition is one of the important mechanisms.

In conclusion, aucuboside can not only alleviate the damaging effects caused by inflammatory cell infiltration in a mouse colitis model but also maintain the balance between Th17 and Treg cells. Further studies are also needed to clarify the detailed mechanisms of how aucuboside inhibits Th17 differentiation in colitis. The results of our study provide a scientific basis for the treatment of IBD using aucuboside and also indicate that aucuboside is one of the main active ingredients of some traditional medicine such as Rehmannia glutinosa in IBD treatment.

\section{DATA AVAILABILITY STATEMENT}

The original contributions presented in the study are included in the article/Supplementary Material, and further inquiries can be directed to the corresponding author.

\section{ETHICS STATEMENT}

The animal study was reviewed and approved by the China Medical University Animal Care and Ethics Committee (CMU2019218), SYXK (Liao) 2018-0008. Written informed consent was obtained from the owners for the participation of their animals in this study.

\section{AUTHOR CONTRIBUTIONS}

CM, XW, FM, XZ, LC, SY, JX, and XS performed the experiments. CM analyzed the data and wrote the paper. YW designed and revised the manuscript.

\section{SUPPLEMENTARY MATERIAL}

The Supplementary Material for this article can be found online at: https://www.frontiersin.org/articles/10.3389/fphar.2021.696599/ full\#supplementary-material 


\section{REFERENCES}

Bassolas-Molina, H., Raymond, E., Labadia, M., Wahle, J., Ferrer-Picón, E., Panzenbeck, M., et al. (2018), An ROR $\gamma$ t Oral Inhibitor Modulates IL-17 Responses in Peripheral Blood and Intestinal Mucosa of Crohn's Disease Patients. Front Immunol. 9, 2307. doi:10.3389/fimmu.2018.02307

Bettelli, E., Carrier, Y., Gao, W., Korn, T., Strom, T. B., Oukka, M., et al. (2006). Reciprocal Developmental Pathways for the Generation of Pathogenic Effector TH17 and Regulatory T Cells. Nature 441 (7090), 235-238. doi:10.1038/ nature 04753

Cao, D., van Vollenhoven, R., Klareskog, L., Trollmo, C., and Malmström, V. (2004). CD25brightCD4+ Regulatory T Cells Are Enriched in Inflamed Joints of Patients with Chronic Rheumatic Disease. Arthritis Res. Ther. 6 (4), R335-R346. doi:10.1186/ar1192

Geremia, A., Biancheri, P., Allan, P., Corazza, G. R., and Di Sabatino, A. (2014). Innate and Adaptive Immunity in Inflammatory Bowel Disease. Autoimmun. Rev. 13 (1), 3-10. doi:10.1016/j.autrev.2013.06.004

Hou, G., and Bishu, S. (2020). Th17 Cells in Inflammatory Bowel Disease: An Update for the Clinician. Inflamm. Bowel Dis. 26 (5), 653-661. doi:10.1093/ibd/izz316

Ivanov, I. I., McKenzie, B. S., Zhou, L., Tadokoro, C. E., Lepelley, A., Lafaille, J. J., et al. (2006). The Orphan Nuclear Receptor ROR $\gamma$ t Directs the Differentiation Program of Proinflammatory IL-17+ T Helper Cells. Cell 126 (6), 1121-1133. doi:10.1016/j.cell.2006.07.035

Ivanov, I. I., Zhou, L., and Littman, D. R. (2007). Transcriptional Regulation of Th17 Cell Differentiation. in Seminars in Immunology. Milan, Italy: Elsevier.

Kolls, J. K., and Lindén, A. (2004). Interleukin-17 Family Members and Inflammation. Immunity 21 (4), 467-476. doi:10.1016/j.immuni.2004.08.018

Korn, T., Bettelli, E., Oukka, M., and Kuchroo, V. K. (2009). IL-17 and Th17 Cells, Annu. Rev. Immunol. 27, 485-517. doi:10.1146/annurev.immunol.021908.132710

Mateus, V., Rocha, J., Mota-Filipe, H., Sepodes, B., and Pinto, R. (2018). Hemin Reduces Inflammation Associated with TNBS-Induced Colitis. Ceg 11 325-334. doi:10.2147/ceg.s166197

McDowell, C., and Haseeb, M. (2019). Inflammatory Bowel Disease (IBD). StatPearls [Internet]. Florida: StatPearls Publishing.

Murakami, S., Tasaka, Y., Takatori, S., Tanaka, A., Kawasaki, H., and Araki, H. (2018). Effect of Eucommia Ulmoides Leaf Extract on Chronic Dextran Sodium SulfateInduced Colitis in Mice. Biol. Pharm. Bull. 41 (6), 864-868. doi:10.1248/bpb.b17-00878

Müzes, G., Molnár, B., Tulassay, Z., and Sipos, F. (2012). Changes of the Cytokine Profile in Inflammatory Bowel Diseases. World J. Gastroenterol. 18 (41), 5848-5861. doi:10.3748/wjg.v18.i41.5848

Nakae, S., Nambu, A., Sudo, K., and Iwakura, Y. (2003). Suppression of Immune Induction of Collagen-Induced Arthritis in IL-17-deficient Mice. J. Immunol. 171 (11), 6173-6177. doi:10.4049/jimmunol.171.11.6173

Rothstein, D. M., and Camirand, G. (2015). New Insights into the Mechanisms of Treg Function. Curr Opin Organ Transplant. 20(4), 376, 384. doi:10.1097/ mot.0000000000000212
Sandquist, I., and Kolls, J. (2018). Update on Regulation and Effector Functions of Th17 Cells. F1000Res 7, 205. doi:10.12688/f1000research.13020.1

Siracusa, R., Fusco, R., Peritore, A. F., Cordaro, M., D’Amico, R., Genovese, T., et al. (2020). The Antioxidant and Anti-inflammatory Properties of Anacardium Occidentale L. Cashew Nuts in a Mouse Model of Colitis. Nutrients 12 (3), 834. doi:10.3390/nu12030834

Smids, C., Horjus Talabur Horje, C. S., Drylewicz, J., Roosenboom, B., Groenen, M. J. M., van Koolwijk, E., et al. (2017). Intestinal T Cell Profiling in Inflammatory Bowel Disease: Linking T Cell Subsets to Disease Activity and Disease Course. J. Crohn's Colitis 12 (4), 465-475. doi:10.1093/ecco-jcc/jjx160

Sugimoto, K., Ogawa, A., Mizoguchi, E., Shimomura, Y., Andoh, A., Bhan, A. K., et al. (2008). IL-22 Ameliorates Intestinal Inflammation in a Mouse Model of Ulcerative Colitis. J. Clin. Invest. 118 (2), 534-544. doi:10.1172/JCI33194

Swiatek, L., and Dombrowicz, E. (1976). Pharmacobotanical Investigations on Some Species of the Scrophulariaceae Family. Part V. Chemical Constitutents in Lathraea Squamaria L. Pol. J. Pharmacol. Pharm. 28 (1), 105-109.

Wang, H., Zhou, X.-M., Wu, L.-Y., Liu, G.-J., Xu, W.-D., Zhang, X.-S., et al. (2020). Aucubin Alleviates Oxidative Stress and Inflammation via Nrf2-Mediated Signaling Activity in Experimental Traumatic Brain Injury. J. Neuroinflammation 17 (1), 188. doi:10.1186/s12974-020-01863-9

Wang, J. Y., Chen, X. J., Zhang, L., Pan, Y. Y., Gu, Z. X., He, S. M., et al. (2018). Comparative Studies of Different Extracts from Eucommia Ulmoides Oliv. Against Rheumatoid Arthritis in CIA Rats. Evid. Based Complement. Alternat Med. 2018, 7379893. doi:10.1155/2018/7379893

Xiong, Y., Wang, J., Chu, H., Chen, D., and Guo, H. (2016). Salvianolic Acid B Restored Impaired Barrier Function via Downregulation of MLCK by microRNA-1 in Rat Colitis Model. Front. Pharmacol. 7 (320). doi:10.3389/ fphar.2016.00134

Yamada, A., Arakaki, R., Saito, M., Tsunematsu, T., Kudo, Y., and Ishimaru, N. (2016). Role of Regulatory T Cell in the Pathogenesis of Inflammatory Bowel Disease. Wjg 22 (7), 2195-2205. doi:10.3748/wjg.v22.i7.2195

Zhang, G., Zhang, C., Sun, J. a., Xiong, Y., Wang, L., and Chen, D. (2021). Phytochemical Regulation of RNA in Treating Inflammatory Bowel Disease and Colon Cancer: Inspirations from Cell and Animal Studies. J. Pharmacol. Exp. Ther. 376 (3), 464-472. doi:10.1124/jpet.120.000354

Conflict of Interest: The authors declare that the research was conducted in the absence of any commercial or financial relationships that could be construed as a potential conflict of interest.

Copyright (c) 2021 Mei, Wang, Meng, Zhang, Chen, Yan, Xue, Sun and Wang. This is an open-access article distributed under the terms of the Creative Commons Attribution License (CC BY). The use, distribution or reproduction in other forums is permitted, provided the original author(s) and the copyright owner(s) are credited and that the original publication in this journal is cited, in accordance with accepted academic practice. No use, distribution or reproduction is permitted which does not comply with these terms. 